\title{
Stratifin indicates worse prognosis and promotes hepatocellular carcinoma proliferation, migration, invasion and EMT by modulating Wnt/ $\beta$-catenin pathway
}

\section{Shanping Ye}

First Affiliated Hospital of Nanchang University

\section{Taiyuan Li}

First Affiliated Hospital of Nanchang University

Jun Shi

First Affiliated Hospital of Nanchang University

Xiaoye Cheng ( $\square$ cxyndyfy@sina.com )

First Affiliated Hospital of Nanchang University https://orcid.org/0000-0002-3436-510X

\section{Research Article}

Keywords: Stratifin, Hepatocellular carcinoma, Prognosis, EMT, Wnt/ $\beta$-catenin pathway.

Posted Date: January 25th, 2022

DOI: https://doi.org/10.21203/rs.3.rs-842346/v2

License: (c) (i) This work is licensed under a Creative Commons Attribution 4.0 International License.

Read Full License 


\section{Abstract}

Purpose: Stratifin (SFN) is closely related to the development of cancers, but the biological role and mechanism of SFN in hepatocellular carcinoma (HCC) remain largely unknown and its function in Wnt/ $\beta$ catenin/EMT pathway in HCC is currently unclear.

Methods: Protein and mRNA expression levels of SFN were detected in HCC and corresponding adjacent non-tumor tissues by western blot and RT-qPCR. The correlation of clinicopathological features and SFN was analyzed. The univariate and multivariate analysis were performed to investigate the prognosis value of SFN in HCC patients based on online database. Artificial modulation of SFN was conducted to explore the role of SFN on HCC cells' proliferation, migration, invasion in vitro, tumor growth in vivo, epithelial mesenchymal transformation (EMT) and activation of Wnt/ $\beta$-catenin signaling.

Results: SFN was up-regulated in HCC cell lines and tissues. Clinically, SFN was positively associated with tumor size, degree of differentiation, TNM stage and vascular invasion. Patients with high SFN levels had worse OS and DFS. Biologically, knockdown of SFN repressed tumor cell proliferation, migration, invasion in vitro and tumor growth in vivo. Conversely, over-expression of SFN promoted these effects. Mechanistically, SFN induced EMT and activated Wnt/ $\beta$-catenin pathway, while depletion of SFN inhibited EMT and activation of Wnt/ $\beta$-catenin pathway.

Conclusions: SFN indicates worse survival in HCC patients and promotes HCC cells' proliferation, migration and invasion. Mechanistically, SFN induces EMT by activating Wnt/ $\beta$-catenin pathway. Our results clarify the role and potential mechanism of SFN in HCC, which may provide a prognostic factor and therapeutic target for HCC patients.

\section{Introduction}

Liver cancer ranks sixth in cancer morbidity and fourth in cancer mortality worldwide in $2018^{[1-3]}$. Hepatocellular carcinoma (HCC) accounts for $75 \%-85 \%$ of primary liver cancer, leading to nearly 745,000 patients deaths in the world every year ${ }^{[1,4]}$, seriously threatening the public health. The incidence of HCC is regionally heterogeneous, about $85 \%$ of HCC patients are estimated to happen in developing or less developed countries, among whom $72 \%$ of patients occur in Asia and more than $50 \%$ cases in China ${ }^{[1,5]}$. Unfortunately, most of HCC patients are at advanced stages when diagnosed and the incidence-tomortality ratio closes to $100 \%{ }^{[6]}$. HCC surveillance (ultrasound and AFP) can help to detect tumor at an early stage when patients are potential to obtain the best curative outcomes ${ }^{[7]}$. For HCC patients, precise evaluation of prognosis is a key element for the treatment decision ${ }^{[8,9]}$. Therefore, it is certainly needed to identify reliable biomarkers for evaluation of prognosis and targets for treatment in $\mathrm{HCC}$ patients ${ }^{[9]}$. Currently, more and more researches indicated that the abnormal expression of genes is closely correlative to the occurrence, development and prognosis of HCC, but the specific functions of most of them are still unclear ${ }^{[10,11]}$. 
Stratifin (SFN) is a checkpoint protein of cell cycle and a regulatory factor of mitotic translation ${ }^{[12]}$. SFN is closely related to many cellular processes, especially for the regulation of cell cycle and signal transduction ${ }^{[13]}$. Overexpression of SFN has been reported in many human tumors, such as ovarian cancer $^{[14]}$, breast cancer ${ }^{[15]}$, lung cancer ${ }^{[12]}, \mathrm{HCC}^{[16]}$. However, downregulation of SFN has also been observed in gliomas ${ }^{[13]}$, nervous system ${ }^{[17]}$, reproductive system ${ }^{[17]}$. Hu et al. have reported that upregulation of SFN was associated with worse prognosis in ovarian cancer ${ }^{[14]}$. Deng et al. indicated that SFN was an independent risk factor of prognosis for patients with gliomas ${ }^{[13]}$. Recently, several studies reported that SFN was up-regulated in $\operatorname{HCC}^{[16,18,19]}$ Song et al. found regulatory effect of SFN on EGFRERK1/2 pathway in HCC ${ }^{[18]}$. However, the biological role and mechanism of SFN in hepatocellular carcinoma (HCC) remains largely unknown and its function in Wnt/ $\beta$-catenin/EMT pathway in HCC is currently unclear.

In this study, we explored the role of SFN in HCC and found that SFN was up-regulated in HCC tissues and cell lines and was positively associated with tumor size, degree of differentiation, TNM stage, vascular invasion, and poor prognosis. SFN promoted HCC proliferation, migration, invasion of HCC cells and tumor growth. Mechanistically, we demonstrated that SFN induced epithelial-to-mesenchymal transition (EMT) and activated Wnt/ $\beta$-catenin pathway. Our results indicated the role and potential mechanism of SFN in HCC, which maight provide a therapeutic target and prognostic marker for HCC.

\section{Material And Methods}

\section{Patients, clinical samples and follow-up}

A total of 34 pairs of HCC tissues and adjacent non-tumor tissues were obtained from HCC patients from the department of General Surgery of the First Affiliated Hospital of Nanchang University. All of the patients underwent radical resection between October 2017 and January 2018. The fresh specimens were stored in liquid nitrogen. The clinical data was collected from electronic medical record and the survival information was obtained by follow-up. The methods of follow-up were similar to the previous research ${ }^{[20]}$. All of the patients did not receive any adjuvant before operation and signed the written informed consent. Our study was compliance with the Helsinki Declaration and was approved by the institutional review of The First Affiliated Hospital of Nanchang University.

\section{Cell lines and cultures}

The HCC cell lines MHCC-97H, MHCC-97L were obtained from the Liver Cancer Institute of Fudan University (Shanghai, China). The HCC cell lines Huh7, SMMC-7721, HepG2 and the normal liver cell line L02 were obtained from the Chinese Academy of Sciences (Shanghai, China). All of them were cultured in Dulbecco's modified Eagle's medium (DMEM, Gibco, USA) containing 10\% fetal bovine serum (FBS, Gibco, USA), $100 \mathrm{U} / \mathrm{ml}$ penicillin and $100 \mathrm{ug} / \mathrm{ml}$ streptomycin (Gibco, USA). All cells were placed in an incubator with $5 \% \mathrm{CO}_{2}$ and humidified at $37^{\circ} \mathrm{C}$. 
Total RNA was obtained from the cultured cells or fresh-frozen tissues by using TRIzol Reagent (Invitrogen, USA) and reverse transcribed into cDNA by using PrimeScript RT reagent Kit with gDNA Eraser (Takara, Japan) in accordance with the manufacturer's instructions. RT-qPCR was performed in a StepOnePlus ${ }^{\mathrm{TM}}$ Real-Time PCR System (Applied Biosystems, USA) by using TB Green ${ }^{\mathrm{R}}$ Premix Ex Taq ${ }^{\mathrm{TM}}$ ॥ (Tli RnaseH Plus) Kit (Takara, Japan). The $\beta$-actin was set as an internal control. All data were analyzed following the method of $2^{-\triangle} \triangle \mathrm{CT}$. The primers used in the current study were as follows: SFN: forward primer: 5'-TGACGACAAGAAGCGCATCAT-3', reverse primer: 5'- GTAGTGGAAGACGGAAAAGTTCA -3'.

\section{Establishment of overexpressing and knockdown HCC cells}

The knockdown and ectopic expression lentiviruses for SFN, and the corresponding control lentivirus were synthesized by HANBIO (Shanghai, China). The two shRNA sequences (names as: shSFN\#1 and shSFN\#2) and cDNA clone (names as: SFN) are presented in the Supplementary Table 1 . The transfection was performed in cell lines according to the manufacturer's protocol. In brief, $1 \times 10^{5} \mathrm{MHCC}$ $97 \mathrm{H}$ or SMMC-7721 cells were seeded in 6-well plates in DMEM with $10 \%$ FBS. The cells were transfected when the cell confluence was about $60 \% .1 \mathrm{ml}$ fresh medium with $20 \mu$ lentivirus solution replaced the old medium. After $4 \mathrm{~h}$, another $1 \mathrm{ml}$ fresh medium added into each well. The medium was replaced after $24 \mathrm{~h}$. The stable transfected cell lines were selected by puromycin with final concentration of 2 $\mathrm{ug} / \mathrm{ml}$. The efficiency of transfection was verified by RT-qPCR and WB.

\section{Cell proliferation assay}

The cell proliferation was assayed using Cell Counting Kit-8 (CCK-8; Dojindo, Japan) according to the protocol of manufacturer. Briefly, $5 \times 10^{3} \mathrm{MHCC}-97 \mathrm{H}$ or SMMC-7721 cells were seeded into 96 -well plates when it stable transfected lentivirus past $48 \mathrm{~h}, 5$ wells for per group. Then, at the time of $0,24,48,72,96$ $\mathrm{h}$, each well was added $10 \mu \mathrm{L}$ CCK-8 reagent, and the cells was incubated in incubator for $2 \mathrm{~h}$. At last, the multimode reader (TECAN SPARK 10M, Switzerland) was used to measure the absorbance at $450 \mathrm{~nm}$.

\section{Colony formation assays}

Infected cells (1000 cells/well in SFN over-expressing group and 500 cells/well in SFN knockdown group respectively) were plated in 6-well plates, and then medium was replaced every five days. After two weeks, the cells were fixed by $4 \%$ paraformaldehyde and stained by $1 \%$ crystal violet. Then, the 6 -plates were washed with running water. The number of cell colony was counted on the pictures.

\section{Wound healing assay}

After transfected $48 \mathrm{~h}, \mathrm{MHCC}-97 \mathrm{H}$ or SMMC-7721 cells were seeded into 6-well plates. When the cells reached to $95 \%$ confluency, the scratch wound was got by a $200 \mu \mathrm{L}$ pipette tip through drawing lines on the surface cells in 6-well plates. The images of wound healing at 0 and $24 \mathrm{~h}$ were photographed using microscope (10x) from each well. 


\section{Migration and invasion assays (Transwell)}

The transwell chambers (Corning, USA) with the pore size at $8 \mu \mathrm{m}$ and 24-well plates were used to assay the cell migration and invasion assays. For the migration experiments, $5 \times 10^{4}$ cells were seeded into the upper chambers in $200 \mu \mathrm{L}$ DMED medium without FBS, while $700 \mu \mathrm{L}$ DMEM containing $20 \%$ FBS was added in the bottom plates. For the invasion experiments, $50 \mu \mathrm{L}$ Matrigel/DMEM (1:8, BD Biosciences, USA) was added into the upper transwell chambers. The other procedures were similar to the migration except for the number of cells $\left(1 \times 10^{5}\right.$ cells). After incubation for $24 \mathrm{~h}$, the chambers were fixed in the $4 \%$ paraformaldehyde and stained with $0.1 \%$ crystal violet. Then, the cells or Matrigel on the upper chambers were removed by cotton swab. HCC cells were counted from five random fields by using microscope (10 $\times)$.

\section{The xenograft mice models}

The 6-week-old male BALB/c nude mice purchased from Hunan SJA Laboratory Animal CO. LTD were used to explore the role of SFN on the tumor growth (six mice for each group). A total of $5 \times 10^{6}$ of HCC cells transfected with lentivirus in 100 ul DMEM medium containing 50\% Matrigel were subcutaneously injected into the right upper flank regions of mice. The tumor length $(L)$ and width $(W)$ were measured every three days. The equation $1 / 2 \times L \times W^{2}$ was used to calculate the tumor volume. After 34 days, the mice were put into the euthanasia box and carbon dioxide was injected into the box at the rate of $30 \%$ of the volume of the euthanasia box per minute. After the animals stopped breathing and its pupils dilated, the next experiments could be carried out. The tumors were excised for further study. All animal researches were approved by the Medical Experimental Animal Care Commission of The First Affiliated Hospital of Nanchang University.

\section{Luciferase reporter assay}

The reporter plasmids encoding TOP-flash or FOP-flash with TCF/LEF DNA binding sites and control plasmids PTK-RL were purchased from Beyotime Biotechnology (China). Infected cells were plated in 24well plates, the pTK-RL and the flash plasmids were co-transfected into the cells. After $48 \mathrm{~h}$, the luciferase activity was analyzed as normalized to Renilla luciferase activity.

\section{Western blotting}

The total proteins from cells and tissues were isolated with RIPA buffer containing protease inhibitors. After centrifugation, the supernatants were collected for subsequently experiments. The NE-PER ${ }^{\mathrm{TM}}$ Nuclear and Cytoplasmic Extraction Reagents (Thermo Scientific, USA) were used to extract nuclear proteins. The BCA Protein Quantitation kit (Pierce, USA) was used to measure the protein concentration. A total of 30 $\mu \mathrm{g}$ protein from cells or tissues were separated by $8 \%-12 \%$ SDS-PAGE and transferred into the polyvinylidene fluoride (PVDF, Millipore, USA) membranes. The PVDF membranes were blocked with $5 \%$ skimmed milk for $1 \mathrm{~h}$ at $37^{\circ} \mathrm{C}$ and incubated with primary antibodies against SFN (ab193667, 1:1000, Abcam), $\beta$-actin (ab8226, 1:1000, Abcam), E-cadherin (\#3195, 1:1000, Cell Signaling Technology), N- 
cadherin(\#13116, 1:1000, Cell Signaling Technology), Vimentin(\#5741, 1:1000, Cell Signaling Technology), MMP-2 (ab92536, 1:1000, Abcam), MMP-9 (ab76003, 1:1000, Abcam), $\beta$-Catenin(\#8480, 1:1000, Cell Signaling Technology), Non-phospho (active) $\beta$-Catenin( $\# 19807,1: 1000$, Cell Signaling Technology), GSK-3ß(\#12456, 1:1000, Cell Signaling Technology), Phospho-GSK-3 $\beta$ (Ser9) (\#5558, 1:1000, Cell Signaling Technology), Axin2(ab109307, 1:1000, Abcam), and c-Myc(ab32072, 1:1000, Abcam) overnight at $4{ }^{\circ} \mathrm{C}$. After washed with Tris-buffered saline Tween buffer, the membranes were incubated with HPR-rabbit (SA00001-2, 1:5000, proteintech) or HPR-mouse (SA00001-1, 1:5000, proteintech) at $37^{\circ} \mathrm{C}$ for $1 \mathrm{~h}$. The intensity of band was quantified by ImageJ (National Institutes of Health, USA) and the specific details were described as previously [21].

\section{SFN expression in TCGA, GEO and Oncomine databases}

The gene expression profiles of liver hepatocellular carcinoma (LIHC) obtained from RNA-Seq HTSeqFPKM platform was downloaded from GDC Data Portal of The Cancer Genome Atlas (TCGA) (https://portal.gdc.cancer.gov/repository) consisted of $374 \mathrm{HCC}$ tissues and 50 adjacent non-tumor tissues. Limma package (R) was used to identify SFN between tumor and non-tumor tissues ${ }^{[22]}$.

For Gene Expression Omnibus (GEO) datasets (https://www.ncbi.nlm.nih.gov/geo/), we downloaded ten expression microarray profiles (GSE: 14520, 25097, 45114, 45436, 55092, 57555, 60502, 76427, 77314, 101728) including HCC tissues and non-tumor tissues. Except for the SFN expression of GSE77314 was directly calculated by the XLSX data downloaded from GEO datasets, difference of SFN expression between tumor and non-tumor tissues of others GEO series were performed by Limma package $(R)^{[22]}$.

For Oncomine database (https://www.oncomine.org/), there were four studies compared SFN expression between hepatocellular carcinoma and normal samples. We comprehensively analyzed four studies with following threshold: $p$-Value $\leq 1 \mathrm{E}-4$, fold change $\geq 2$, gene ranks in the top $10 \%$.

\section{Prognosis Analysis based on online database}

To investigate the prognosis value of the SFN gene in patients with HCC, we conducted survival analysis and univariate and multivariate analysis. The univariate and multivariate Cox regression analysis of OS were performed by "survival" and "survminer" packages (https://cran.r-project.org/web/packages/) based on $\mathrm{R}$ language. The data downloaded from Kaplan-Meier plotter database (http://kmplot.com/analysis/) was used for survival analysis ${ }^{[23]}$. Graphpad prism software (Version 7) was used to plot all survival curves.

\section{Statistical analysis}

The data statistical analyses in this paper were performed using SPSS 22.0 software and GraphPad Prism 7. Mann-Whitney U-test, student $t$ test or Chi-square test, and Spearman's rank analysis was performed according to the type of variables. A two-tailed $P \otimes 0.05$ were considered as statistically significant. 


\section{Results}

\section{SFN is highly expressed in human HCC cell lines and tissues}

To determine the expression level of SFN in HCC, we detected the expression levels of SFN in the HCC cell lines and normal cell L02. Both SFN mRNA and protein was up-regulated in all HCC cell lines compared to L02 cell (Fig. 1A and 1C). Furthermore, we examined the SFN mRNA and protein levels in clinical fresh HCC tissues. The HCC tissues had higher mRNA and protein expression levels of SFN than that in adjacent nontumor liver tissues (Fig. 1B and 1D). To further confirm the level of SFN expression, we also analyzed data derived from the TCGA, GEO and Oncomine database. As shown in Fig. 1E the SFN mRNA based on TCGA was significantly up-regulated in HCC tissues ( $P \square 0.05)$. In Oncomine database, the SFN gene was also obviously overexpressed in HCC tissues in Wurmbach Liver dataset, Mas Liver dataset, Roessler Liver 2 dataset, Roessler Liver dataset (all $P \otimes 0.05$, Fig. 1F-1I). As shown in Supplement Fig. 1A, the high SFN expression was also validated from the meta-analysis of four Oncomine analyses $(P<$ 0.05). Finally, we analyzed the expression of SFN in GEO databases. Consistent with the results of TCGA and Oncomine database, the SFN gene was also up-regulated in ten GEO series (GSE: 14520, 25097, 45114, 45436, 55092, 57555, 60502, 76427, 77314, 101728, shown in Supplement Fig. 1B-1K, all $P$ $\triangle 0.05)$.

Based on these results, we concluded that SFN was significantly up-regulated in human HCC cell lines and tissues.

\section{SFN was closely associated with aggressive clinicopathological features and poor prognosis in HCC patients}

To further study the clinicopathological significance of SFN, we analyzed the correlation between SFN expression and clinicopathological characteristics in $34 \mathrm{HCC}$ patients from our hospital. Patients were divided into high and low SFN expression groups based on the median expression level of SFN mRNA. The SFN expression is bound up with the tumor size, degree of differentiation, TNM stage, and vascular invasion $(P \otimes 0.05$, Table 1$)$.

To evaluate the prognostic value of SFN in HCC patients, we compared the differences of overall survival (OS) and disease-free survival (DFS) between high and low SFN group in 34 HCC patients. As we expected, SFN high group was associated with worse 3 years OS and DFS as compared with low SFN group ( $P \otimes 0.05$, Fig. 2A-2B). We further performed survival analysis using the Kaplan-Meier plotter online database. The results indicated that SFN over-expression in HCC tumor was significantly associated with worse OS, progression-free survival (PFS), relapse-free survival (RFS), and disease-free survival (DFS) in HCC patients ( all log rank $P \otimes 0.05$, Fig. 2C-2F).

Furthermore, the clinicopathological data of 374 cases of HCC obtained from TCGA data were used to evaluate the prognostic value of SFN in HCC cases by univariate and multivariate analysis. Due to the incomplete clinicopathological data of many cases, we selected some common features (age, gender, 
tumor grade, TNM stage) for further analysis and excluded unavailable data including unknown, missing, Tx, Nx and Mx data. Finally, 212 HCC patients were enrolled in this study. The univariate analysis indicated that TNM stage, T, M, SFN expression were significantly associated with OS $(P=6.71 \mathrm{e}-07$, $5.65 \mathrm{e}-07,0.0227$ and 3.02e-06, respectively, Table 2). As shown in Table 2, multivariable analysis indicated that SFN was an independent prognostic factor for OS $(P=0.0005)$.

In conclusion, our results suggested that SFN was closely associated with aggressive clinicopathological features and poor prognosis in HCC patients.

\section{SFN promotes HCC cells proliferation, migration, invasion in vitro and tumor growth in vivo}

To explore the biological function of SFN in the progress of HCC, we up regulated SFN expression by ectopic expression lentiviruses in the SMMC-7721 (named: SMMC-7721-Lv-SFN) cell with the lowest SFN expression, and we down-regulated SFN expression by knockdown expression lentiviruses in the MHCC97H (named: MHCC-97H-Lv-shSFN) cell with the highest SFN expression. The RT-qPCR and western blotting were used to estimate the knockdown efficiency (Fig. 3A-3D). CCK-8 and colony formation assays were used to explore the proliferative capacity of two kinds of cell lines. We found that the overexpression of SFN promoted colony formation and proliferation ability (all $P \otimes 0.05$, Fig. 3I-3L). Conversely, SFN knockdown inhibited colony formation and proliferation ability (all $P \otimes 0.05$, Fig. 3l-3L). Furthermore, to identify the function of SFN on invasion and migration of HCC cells, we conducted transwell assay and wound-healing. In wound-healing experiments, we observed that the cells with SFN knockdown presented a obvious reduction in wound formation capacity compared with the control cells, while SFN over-expression enhanced migration ability (Fig. 3E-3F). Similarly, transwell assays displayed SFN depletion significantly decreased the migration and invasion ability of HCC cells, while SFN overexpression increased the cells' migration and invasion ability compared with the control group (Fig. 3G$3 \mathrm{H})$. Importantly, we evaluated the effect of SFN on tumor growth in vivo. In line with our results in vitro, we found that the SMMC-7721-Lv-SFN cells obviously enhanced the growth of tumor compared with SMMC-7721-control cells. The MHCC-97H-Lv-shSFN cells significantly inhibited the abilities of tumor growth (Fig. 3M). The tumor weight of the SMMC-7721-Lv-SFN cells were markedly higher than that of SMMC-7721-control cells(Fig. 30) while the tumor weight of MHCC-97H-Lv-shSFN cells were significantly lower than that of MHCC-97H-control cells (Fig. 3N).

Taken together, these data demonstrated that SFN promoted HCC cells proliferation, migration, invasion in vitro and tumor growth in vivo.

\section{SFN induces EMT and activates Wnt/ $\beta$-catenin signaling}

EMT (epithelial-mesenchymal transition) is an important process for tumor progression. To further explore the mechanism of SFN in HCC progression, we examined the markers of EMT. We found that the ectopic expression of SFN in SMMC-7721 resulted in increased expression of N-cadherin and Vimentin (mesenchymal markers) and decreased expression of E-cadherin (epithelial marker), while SFN knockdown in MHCC-97H cells led to the opposite results as shown by western blot (Fig. 4A and 4B). 
MMP2 and MMP9, the members of the matrix metalloproteinase family, are closely associated with cell migration and invasion. We detected MMP2 and MMP9 and found that MMP-2, MMP-9 were up-regulated in SFN-over-expressing cells and down-regulated in SFN knockdown cells (Fig. 4A and 4B). As we known, Wnt/ $\beta$-catenin signaling is a crucial pathway for HCC progression and EMT can be induced by activation of $W n t / \beta$-catenin signaling ${ }^{[24,25]}$. Therefore, we speculated that SFN may activate $W n t / \beta$-catenin signaling to induce EMT and promote HCC progression. To verify the hypothesis, we explored the role of SFN on the expression of key markers, $\beta$-catenin and GSK-3 $\beta$, of the Wnt/ $\beta$-catenin signaling pathway ${ }^{[26,27]}$. In SFN-over-expressing cells, we observed that $\beta$-catenin, non-phospho (active) $\beta$-catenin,

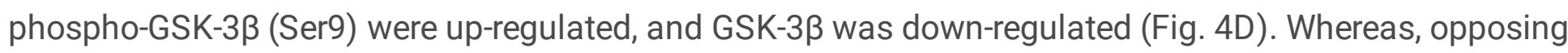
results were obtained from the SFN knockdown cells (Fig. 4C). As we known, the translocation into nuclear of $\beta$-catenin is the best biomarker for activation of Wnt/ $\beta$-catenin signaling ${ }^{[28]}$. We also detected the protein expression levels of $\beta$-catenin in nuclear and found that SFN over-expression promoted $\beta$ catenin accumulation in nuclear and SFN knockdown exhibited contrary trend (Fig. 4E-4F). Additionally, we used the TOP/FOP-Flash test to further confirm the activity of Wnt/ $\beta$-catenin signaling. The TOP/FOPFlash reporter assays indicated that down-regulation or over-expression of SFN obviously enhanced or repressed activity of Wnt/ $\beta$-catenin signaling (Fig. 4G-4H). Finally, to further clarify that SFN induced EMT through Wnt/ $\beta$-catenin signaling, we performed the experiments in SMMC-7721-Lv-SFN cells by using XAV-939, a widely used inhibitor of Wnt/ $\beta$-catenin signaling ${ }^{[29]}$. As shown in Fig. 4I-4J, EMT was inhibited after Wnt/ $\beta$-catenin signaling being repressed by XAV-939 in MHCC-97H cells with SFN overexpression.

Taken together, the above results indicated that SFN induces EMT and activates Wnt/ $\beta$-catenin signaling. SFN regulated EMT by Wnt/ $\beta$-catenin signaling.

\section{Discussion}

HCC, a life-threatening cancer, is the common cause of cancer-related death around the world, particularly in the Asia-Pacific region ${ }^{[30]}$. $\mathrm{HCC}$ is also a cancer with heterogeneous feature and the clinical outcome is variable after treatment. Over the past few years, considerable researches have been performed to investigate prognostic markers for $\mathrm{HCC}^{[31]}$. It has been reported that the high level of C-reactive protein at the time of diagnosis was associated with worse survival of HCC patients ${ }^{[32]}$. In our study, we find that the expression of SFN is increased in HCC and significantly related to aggressive clinicopathological features and worse survival of patients with HCC. Moreover, our results indicate that SFN plays important roles in HCC proliferation, migration, invasion, tumor growth and induces EMT by activating Wnt/ $\beta$ catenin signaling. Therefore, SFN may be a novel oncogene in $\mathrm{HCC}$ and is expected to be a therapeutic target and prognostic factor for patients with HCC.

SFN is a member from conserved protein family of 14-3-3 and plays important functions in various biological processes ${ }^{[3]}$. Over-expression of SFN has been reported in human malignant tumors. SFN is also a prognostic factor for many malignant tumors, such as ovarian cancer ${ }^{[14]}$, gliomas ${ }^{[13]}$ and 
gallbladder cancer ${ }^{[34]}$. In our study, SFN was up-regulated in HCC patients and associated with aggressive clinicopathological features. Moreover, we observed that patients with high SFN expression were related with worse survival than that with low SFN expression. We further validated the result in public databases. The TCGA, GEO, and Oncomine databases greatly promoted cancer research due to containing much information of gene expression and clinical data ${ }^{[35-37]}$. As shown in the Figure $2 A$ to $2 \mathrm{~J}$, the gene expression data in GEO database showed the higher expression of SFN in HCC than that in non-tumor tissues. Our results were consistent with previous studies ${ }^{[38,39]}$. What's more, we investigated the prognosis value by using the Kaplan-Meier plotter online database and found that SFN overexpression in HCC tumor was significantly associated with worse OS, PFS, RFS, and DSS in HCC patients. Our data suggested that SFN promoted HCC prognosis. Previous researches have referred to the potential role of SFN in tumors ${ }^{[40,41]}$.

SFN was closely correlated with cell cycle, apoptosis, signal transduction and protein trafficking ${ }^{[42]}$. Our results of functional experiments demonstrated that over-expression of SFN obviously promoted HCC cells' proliferation, migration and invasion, while SFN knockdown in HCC cells displayed opposing trend. Our study also revealed that SFN could enhance the tumor growth in vivo. EMT is vital in cancer development and progression ${ }^{[43]}$. EMT helps tumor cells acquire mesenchymal phenotype and ability of migration and invasion ${ }^{[43]}$. Our results indicated that depletion of SFN suppressed the process of EMT while over-expression of SFN promoted it. Our data further enriched the understanding of the biological role of SFN in HCC progression.

In the current study, we further illustrated that SFN facilitated HCC cells proliferation, migration, invasion, tumor growth, and EMT by activation of Wnt/ $\beta$-catenin signaling. Wnt/ $\beta$-catenin signaling played an important role in progression of $\mathrm{HCC}{ }^{[44]}$. $\beta$-catenin and Gsk-3 $\beta$ are key components of Wnt/ $\beta$-catenin signaling pathway ${ }^{[27,44]}$. As we known, the levels of stable $\beta$-catenin play a key function in the output $W n t / \beta$-catenin signal ${ }^{[26]}$. Our results showed that the protein expression levels of $\beta$-catenin, non-phospho (active) $\beta$-catenin, and phospho-GSK-3 $\beta$ (Ser9) in SFN-over-expressing cells were up-regulated. SFN knockdown showed contrary results. To further confirm the function of SFN on Wnt/ $\beta$-catenin pathway, we examined the protein level of $\beta$-catenin in nuclear and found that SFN over-expression promoted accumulation of $\beta$-catenin in nuclear and SFN knockdown exhibited contrary trend. These results may help us understand the relationship of SFN and Wnt/ $\beta$-catenin signaling. However, this is just a preliminary exploration and deeper mechanism need to be further investigated.

\section{Conclusions}

In conclusion, SFN is over-expressed in HCC tissues and cell lines and is correlated with tumor size, differentiation, vascular invasion and TNM stage. The high expression of SFN was obviously associated with worse survival. SFN promotes cell proliferation, migration, invasion and tumor growth and maybe an oncogene in HCC. Moreover, SFN induces EMT and activates the Wnt/ $\beta$-catenin signaling pathway. The 
results of current analysis may provide a prognostic factor and therapeutic target for patients with HCC in future.

\section{Declarations}

\section{Funding}

The authors declare that no funds, grants, or other support were received during the preparation of this manuscript.

\section{Competing Interests}

The authors declare that they have no financial or non-financial interests to disclose.

\section{Author Contributions}

Shanping Ye and Xiaoye Cheng designed the study, conducted the experiments and collected data; Shanping Ye, Xiaoye Cheng, Taiyuan Li and Jun Shi analyzed the data; Shanping Ye wrote the manuscript; Xiaoye Cheng, Shi Jun, Taiyuan Li proofread and revised the manuscript. All authors red and approved the final manuscript.

\section{Ethics approval}

The patients study protocol was approved by the institutional review of The First Affiliated Hospital of Nanchang University and the study compliance with the Helsinki Declaration. All animal researches were approved by the Medical Experimental Animal Care Commission of The First Affiliated Hospital of Nanchang University.

\section{Consent to participate}

Informed consent was obtained from all individual participants included in the study.

\section{Consent to publish}

The authors affirm that human research participants provided informed consent for publication of the images in Figure 1B and 1D.

\section{Data Availability}

The gene expression profiles of liver hepatocellular carcinoma (LIHC) obtained from RNA-Seq HTSeqFPKM platform were downloaded from GDC Data Portal of The Cancer Genome Atlas (TCGA) (https://portal.gdc.cancer.gov/repository) .The GSE datasets (GSE: 14520, 25097, 45114, 45436, 55092, $57555,60502,76427,77314,101728)$ were downloaded from GEO databases (https://www.ncbi.nlm.nih.gov/geo/), 
The Oncomine database (https://www.oncomine.org/) was used for meta-analysis of SFN expression. Access to the database can be obtained from the corresponding author on reasonable request.

\section{References}

1. Bray F, Ferlay J, Soerjomataram I, et al. Global cancer statistics 2018: GLOBOCAN estimates of incidence and mortality worldwide for 36 cancers in 185 countries. CA Cancer J Clin. 2018;68(6):394-424. DOI:10.3322/caac.21492]. [PMID: 30207593.

2. Wang $\mathrm{H}$, Naghavi $\mathrm{M}$, Allen $\mathrm{C}$, et al. Global, regional, and national life expectancy, all-cause mortality, and cause-specific mortality for 249 causes of death, 1980-2015: a systematic analysis for the Global Burden of Disease Study 2015. The Lancet. 2016;388(10053):1459-544. [DOI: 10.1016/s0140-6736(16)31012-1].

3. Villanueva A. Hepatocellular Carcinoma. N Engl J Med. 2019;380(15):1450-62. [PMID: 30970190 DOI: 10.1056/NEJMra1713263].

4. Yang JD, Roberts LR. Hepatocellular carcinoma: A global view. Nat Rev Gastroenterol Hepatol. 2010;7(8):448-58. DOI:10.1038/nrgastro.2010.100]. [PMID: 20628345.

5. Tang A, Hallouch O, Chernyak V, Kamaya A, Sirlin CB. Epidemiology of hepatocellular carcinoma: target population for surveillance and diagnosis. Abdom Radiol (NY). 2018;43(1):13-25. DOI:10.1007/s00261-017-1209-1]. [PMID: 28647765.

6. Singal AG, Lampertico P, Nahon P. Epidemiology and surveillance for hepatocellular carcinoma: New trends. J Hepatol. 2020;72(2):250-61. DOI:10.1016/j.jhep.2019.08.025]. [PMID: 31954490.

7. Singal AG, Pillai A, Tiro J. Early detection, curative treatment, and survival rates for hepatocellular carcinoma surveillance in patients with cirrhosis: a meta-analysis. PLoS Med 2014; 11(4): e1001624 [PMID: 24691105 DOI: 10.1371/journal.pmed.1001624].

8. Zhang B, Finn RS. Personalized Clinical Trials in Hepatocellular Carcinoma Based on Biomarker Selection. Liver Cancer. 2016;5(3):221-32. [PMID: 27493897 DOI: 10.1159/000367763].

9. Black AP, Mehta AS. The search for biomarkers of hepatocellular carcinoma and the impact on patient outcome. Curr Opin Pharmacol. 2018;41:74-8. DOI:10.1016/j.coph.2018.04.002]. [PMID: 29772420.

10. Ding ZY, Jin GN, Wang W, et al. Reduced expression of transcriptional intermediary factor 1 gamma promotes metastasis and indicates poor prognosis of hepatocellular carcinoma. Hepatology. 2014;60(5):1620-36. [PMID: 24954480 DOI: 10.1002/hep.27273].

11. Xia L, Huang W, Tian D, et al. Upregulated FoxM1 expression induced by hepatitis $B$ virus $X$ protein promotes tumor metastasis and indicates poor prognosis in hepatitis B virus-related hepatocellular carcinoma. J Hepatol. 2012;57(3):600-12. DOI:10.1016/j.jhep.2012.04.020]. [PMID: 22613004.

12. Kim Y, Shiba-Ishii A, Nakagawa T, et al. Stratifin regulates stabilization of receptor tyrosine kinases via interaction with ubiquitin-specific protease 8 in lung adenocarcinoma. Oncogene. 2018;37(40):5387-402. DOI:10.1038/s41388-018-0342-9]. [PMID: 29880877. 
13. Deng J, Gao G, Wang L, et al. Stratifin expression is a novel prognostic factor in human gliomas. Pathol Res Pract. 2011;207(11):674-9. DOI:10.1016/j.prp.2011.08.005]. [PMID: 21940111.

14. Hu Y, Zeng Q, Li C, Xie Y. Expression profile and prognostic value of SFN in human ovarian cancer. Biosci Rep 2019; 39(5) [PMID: 30926680 DOI: 10.1042/BSR20190100].

15. Ko S, Kim JY, Jeong J, et al. The role and regulatory mechanism of 14-3-3 sigma in human breast cancer. J Breast Cancer. 2014;17(3):207-18. DOI:10.4048/jbc.2014.17.3.207]. [PMID: 25320618.

16. Zhang Y, Li Y, Lin C, et al. Aberrant upregulation of 14-3-3sigma and EZH2 expression serves as an inferior prognostic biomarker for hepatocellular carcinoma. PLoS One. 2014;9(9):e107251. DOI:10.1371/journal.pone.0107251]. [PMID: 25226601.

17. Winter M, Lodygin D, Verdoodt B, Hermeking H. Deletion of 14-3-3sigma sensitizes mice to DMBA/TPA-induced papillomatosis. Oncotarget. 2016;7(30):46862-70. DOI:10.18632/oncotarget.10478]. [PMID: 27409835.

18. Song J, Liu Y, Liu F, et al. The 14-3-3sigma protein promotes HCC anoikis resistance by inhibiting EGFR degradation and thereby activating the EGFR-dependent ERK1/2 signaling pathway. Theranostics 2021; 11(3): 996-1015 [PMID: 33391517 DOI: 10.7150/thno.51646].

19. Wu YJ, Jan YJ, Ko BS, Liang SM, Liou JY. Involvement of 14-3-3 Proteins in Regulating Tumor Progression of Hepatocellular Carcinoma. Cancers (Basel). 2015;7(2):1022-36. [PMID: 26083935 DOI: 10.3390/cancers7020822].

20. Zhong X, Kan A, Zhang W, et al. CBX3/HP1gamma promotes tumor proliferation and predicts poor survival in hepatocellular carcinoma. Aging. 2019;11(15):5483-97. [PMID: 31375643 DOI: 10.18632/aging.102132].

21. Qu W, Wen X, Su K, Gou W. MiR-552 promotes the proliferation, migration and EMT of hepatocellular carcinoma cells by inhibiting AJAP1 expression. J Cell Mol Med. 2019;23(2):1541-52.

DOI:10.1111/jcmm.14062]. [PMID: 30597727.

22. Ritchie ME, Phipson $B$, Wu D, et al. limma powers differential expression analyses for RNAsequencing and microarray studies. Nucleic Acids Res 2015; 43(7): e47 [PMID: 25605792 DOI: 10.1093/nar/gkv007].

23. Menyhart O, Nagy A, Gyorffy B. Determining consistent prognostic biomarkers of overall survival and vascular invasion in hepatocellular carcinoma. R Soc Open Sci. 2018;5(12):181006. DOI:10.1098/rsos.181006]. [PMID: 30662724.

24. Teeuwssen M, Fodde R. Wnt Signaling in Ovarian Cancer Stemness, EMT, and Therapy Resistance. J Clin Med 2019; 8(10) [PMID: 31614568 DOI:10.3390/jcm8101658].

25. Gonzalez DM, Medici D. Signaling mechanisms of the epithelial-mesenchymal transition. Sci Signal 2014; 7(344): re8 [PMID: 25249658 DOI: 10.1126/scisignal.2005189].

26. Clevers H, Nusse R. Wnt/beta-catenin signaling and disease. Cell 2012; 149(6): 1192-1205 [PMID: 22682243 DOI: 10.1016/j.cell.2012.05.012].

27. Russell JO, Monga SP. Wnt/beta-Catenin Signaling in Liver Development, Homeostasis, and Pathobiology. Annu Rev Pathol. 2018;13:351-78. DOl:10.1146/annurev-pathol-020117-044010]. 
[PMID: 29125798.

28. Zhang T, Ma Z, Liu L, et al. DDX39 promotes hepatocellular carcinoma growth and metastasis through activating Wnt/beta-catenin pathway. Cell Death Dis. 2018;9(6):675. DOI:10.1038/s41419018-0591-0]. [PMID: 29867138.

29. Zhang J, Qin M, Yang D, et al. Nanoprotein Interaction Atlas Reveals the Transport Pathway of Gold Nanoparticles across Epithelium and Its Association with Wnt/beta-Catenin Signaling. ACS Nano 2021 [PMID: 34672537 DOI:10.1021/acsnano.1c06452].

30. Zhu RX, Seto WK, Lai CL, Yuen MF. Epidemiology of Hepatocellular Carcinoma in the Asia-Pacific Region. Gut Liver. 2016;10(3):332-9. DOI:10.5009/gnl15257]. [PMID: 27114433.

31. Burkhart RA, Ronnekleiv-Kelly SM, Pawlik TM. Personalized therapy in hepatocellular carcinoma: Molecular markers of prognosis and therapeutic response. Surg Oncol. 2017;26(2):138-45. DOI:10.1016/j.suronc.2017.01.009]. [PMID: 28577719.

32. Sieghart W, Pinter M, Hucke F, et al. Single determination of C-reactive protein at the time of diagnosis predicts long-term outcome of patients with hepatocellular carcinoma. Hepatology. 2013;57(6):2224-34. [DOI: 10.1002/hep.26057].

33. Chan TA, Hermeking $H$, Lengauer $C$, Kinzler KW, Vogelstein B. 14-3-3Sigma is required to prevent mitotic catastrophe after DNA damage. Nature. 1999;401(6753):616-20. [PMID: 10524633 DOI: 10.1038/44188].

34. Sirivatanauksorn V, Dumronggittigule W, Dulnee B, et al. Role of stratifin (14-3-3 sigma) in adenocarcinoma of gallbladder: A novel prognostic biomarker. Surg Oncol. 2020;32:57-62. DOI:10.1016/j.suronc.2019.10.022]. [PMID: 31751820.

35. Hutter C, Zenklusen JC. The Cancer Genome Atlas: Creating Lasting Value beyond Its Data. Cell. 2018;173(2):283-5. DOI:10.1016/j.cell.2018.03.042]. [PMID: 29625045.

36. Chen Y, Li Y, Narayan R, Subramanian A, Xie X. Gene expression inference with deep learning. Bioinformatics. 2016;32(12):1832-9. D0I:10.1093/bioinformatics/btw074]. [PMID: 26873929.

37. Rhodes DR, Yu J, Shanker K, et al. ONCOMINE: a cancer microarray database and integrated datamining platform. Neoplasia. 2004;6(1):1-6. [PMID: 15068665 DOI: 10.1016/s1476-5586(04)800472].

38. Reis H, Putter C, Megger DA, et al. A structured proteomic approach identifies 14-3-3Sigma as a novel and reliable protein biomarker in panel based differential diagnostics of liver tumors. Biochim Biophys Acta. 2015;1854(6):641-50. DOl:10.1016/j.bbapap.2014.10.024]. [PMID: 25448011.

39. Liu CC, Jan YJ, Ko BS, et al. 14-3-3sigma induces heat shock protein 70 expression in hepatocellular carcinoma. BMC Cancer. 2014;14:425. DOI:10.1186/1471-2407-14-425]. [PMID: 24923353.

40. Huang Y, Yang M, Huang W. 14-3-3 sigma: A potential biomolecule for cancer therapy. Clin Chim Acta. 2020;511:50-8. DOI:10.1016/j.cca.2020.09.009]. [PMID: 32950519.

41. Neal CL, Yu D. 14-3-3zeta as a prognostic marker and therapeutic target for cancer. Expert Opin Ther Targets. 2010;14(12):1343-54. DOI:10.1517/14728222.2010.531011]. [PMID: 21058923. 
42. Smith AJ, Daut J, Schwappach B. Membrane proteins as 14-3-3 clients in functional regulation and intracellular transport. Physiology (Bethesda). 2011;26(3):181-91. DOI:10.1152/physiol.00042.2010]. [PMID: 21670164.

43. Dongre A, Weinberg RA. New insights into the mechanisms of epithelial-mesenchymal transition and implications for cancer. Nat Rev Mol Cell Biol. 2019;20(2):69-84. DOI:10.1038/s41580-018-0080-4]. [PMID: 30459476.

44. Perugorria MJ, Olaizola P, Labiano I, et al. Wnt-beta-catenin signalling in liver development, health and disease. Nat Rev Gastroenterol Hepatol. 2019;16(2):121-36. DOI:10.1038/s41575-018-0075-9]. [PMID: 30451972.

\section{Tables}

Table 1 Clinicopathological features of 34 HCC patients between SFN high and low expression cohorts 


\begin{tabular}{|c|c|c|c|}
\hline \multirow[t]{2}{*}{ Features } & \multicolumn{2}{|l|}{ qRT-PCR } & \multirow[t]{2}{*}{$P$ value } \\
\hline & $\operatorname{Low}(n=17)$ & High $(n=17)$ & \\
\hline Gender, n & & & 0.656 \\
\hline Male & 15 & 13 & \\
\hline Female & 2 & 4 & \\
\hline Age, years & & & 0.732 \\
\hline$\leq 50$ & 7 & 9 & \\
\hline$\otimes 50$ & 10 & 8 & \\
\hline HBV infection, $n$ & & & 1.000 \\
\hline Positive & 16 & 15 & \\
\hline Negtive & 1 & 2 & \\
\hline AFP, ng/ml & & & 0.166 \\
\hline$\leq 400$ & 7 & 12 & \\
\hline$\otimes 400$ & 10 & 5 & \\
\hline Tumor number, $\mathrm{n}$ & & & 1.000 \\
\hline Solitary & 16 & 15 & \\
\hline Multiple & 1 & 2 & \\
\hline Tumor size, cm & & & $0.013^{*}$ \\
\hline$\leq 5$ & 14 & 6 & \\
\hline$\otimes 5$ & 3 & 11 & \\
\hline Vascular invasion, $\mathrm{n}$ & & & $0.037 *$ \\
\hline Yes & 4 & 11 & \\
\hline None & 13 & 6 & \\
\hline Tumor differentiation, $\mathrm{n}$ & & & $0.003^{*}$ \\
\hline Well/moderate & 17 & 9 & \\
\hline Poor & 0 & 8 & \\
\hline Liver cirrhosis, $\mathrm{n}$ & & & 0.708 \\
\hline Yes & 13 & 11 & \\
\hline None & 4 & 6 & \\
\hline
\end{tabular}

Page 16/23 


\begin{tabular}{|lccc|}
\hline AJCC TNM stage, $\mathrm{n}$ & & & $\mathbf{0 . 0 1 3}^{*}$ \\
\hline I/ II & 14 & 6 & \\
\hline III/ IV & 3 & 11 & \\
\hline
\end{tabular}

AFP: Alpha-fetoprotein; AJCC: American Joint Committee on Cancer; HBV: Hepatitis B virus; HCC.

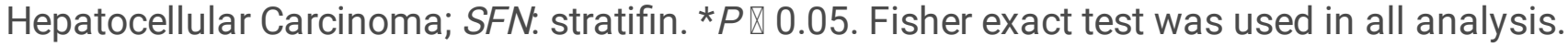

Table 2 Univariate and multivariate survival analysis of OS in HCC patients from online databases

\begin{tabular}{|lllllll|}
\hline Variable & \multicolumn{2}{l}{ Univariate analysis } & \multirow{2}{*}{$P$ value } & \multicolumn{2}{l}{ Multivariate analysis } & $P$ value \\
\cline { 2 - 3 } & HR & $95 \% \mathrm{Cl}$ & & HR & $95 \% \mathrm{Cl}$ & \\
Age & 1.006 & $0.987-1.025$ & 0.538 & 1.012 & $0.991-1.033$ & 0.275 \\
\hline Gender & 0.845 & $0.516-1.385$ & 0.505 & 1.022 & $0.585-1.787$ & 0.938 \\
\hline Grade & 0.996 & $0.726-1.366$ & 0.979 & 1.159 & $0.821-1.636$ & 0.403 \\
AJCC TNM & 1.921 & $1.485-2.485$ & $\mathbf{0 . 0 0 0}$ & 2.041 & $0.642-6.488$ & 0.227 \\
\hline T(tumor) & 1.844 & $1.451-2.344$ & $\mathbf{0 . 0 0 0}$ & 0.937 & $0.320-2.745$ & 0.906 \\
\hline N(node) & 2.018 & $0.492-8.279$ & 0.330 & 1.080 & $0.125-9.310$ & 0.944 \\
\hline M(metastasis) & 3.867 & $1.208-12.374$ & $\mathbf{0 . 0 2 3}$ & 0.755 & $0.191-2.987$ & 0.689 \\
\hline SFN expression & 1.009 & $1.005-1.013$ & $\mathbf{0 . 0 0 0}$ & 1.008 & $1.004-1.013$ & $\mathbf{0 . 0 0 0}$ \\
\hline
\end{tabular}

Cl: Confidence interval; HCC. Hepatocellular carcinoma; HR: Hazard ratio; OS. Overall survival; SFN: Stratifin.

\section{Figures}



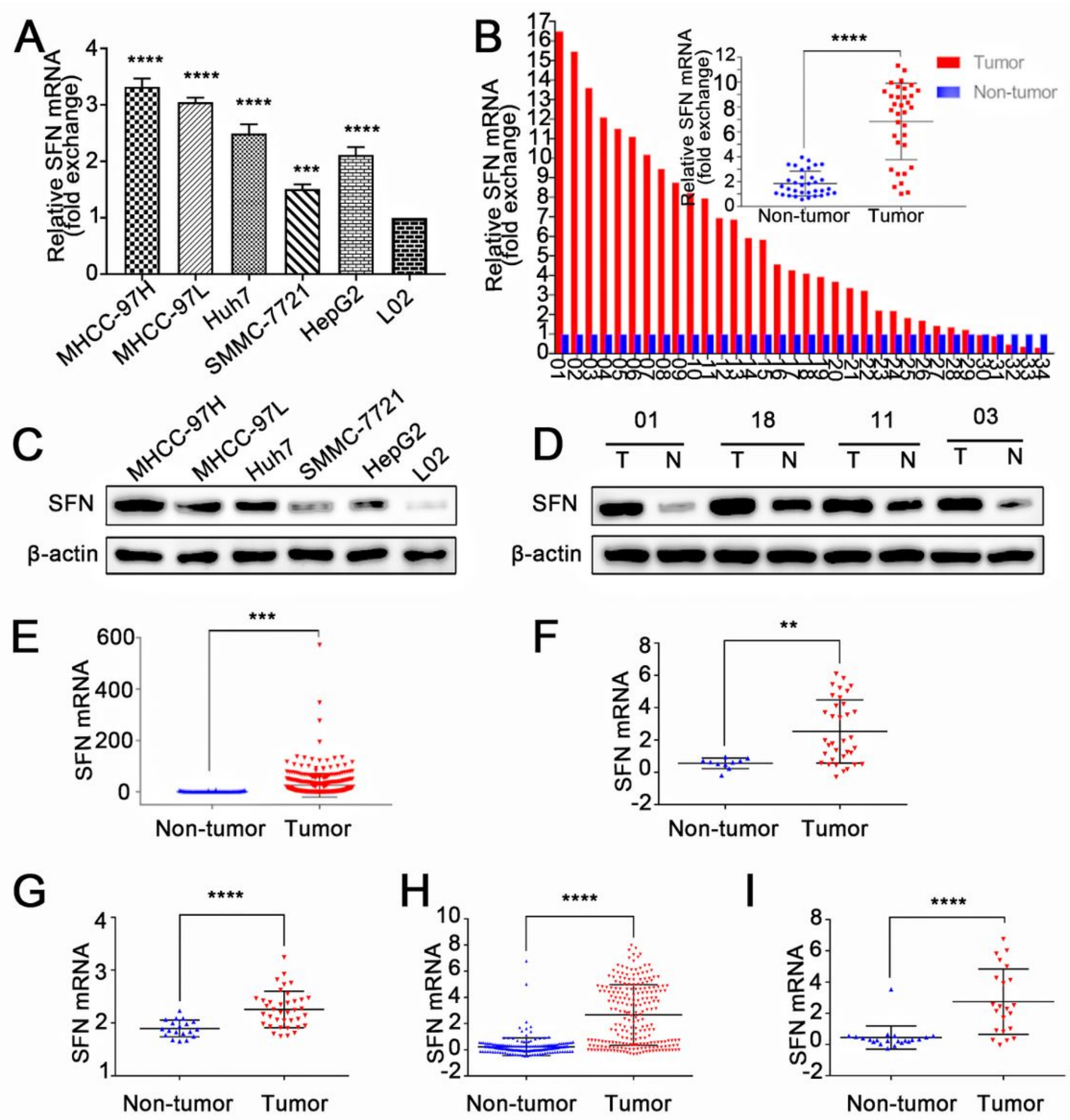

Figure 1

SFN gene expression is obviously up-regulated in HCC cells and tissues

(A) SFN mRNA was detected in five kinds of HCC cell lines and normal liver cells (L02) by RT-qPCR. (B) SFN mRNA was measured in 34 paired fresh HCC tissues and adjacent non-tumor tissues by RT-qPCR. (C) SFN mRNA was detected in five kinds of HCC cell lines and normal liver cells (LO2) by western 
blotting. (D) Representative images of western blot in 34 paired fresh HCC tissues and adjacent nontumor tissues. (E) SFN mRNA in 377 HCC cases compared to 50 non-tumor cases by analyzing TCGA dataset. (F-I) SFN mRNA expression in HCC tissues in four Oncomine datasets (Roessler liver, Mas liver, Roessler liver 2, Wurmbach liver). ${ }^{* \star} \mathrm{P} \otimes 0.01,{ }^{* \star \star} \mathrm{P} \otimes 0.001,{ }^{* \star \star *} \mathrm{P} \otimes 0.0001$.
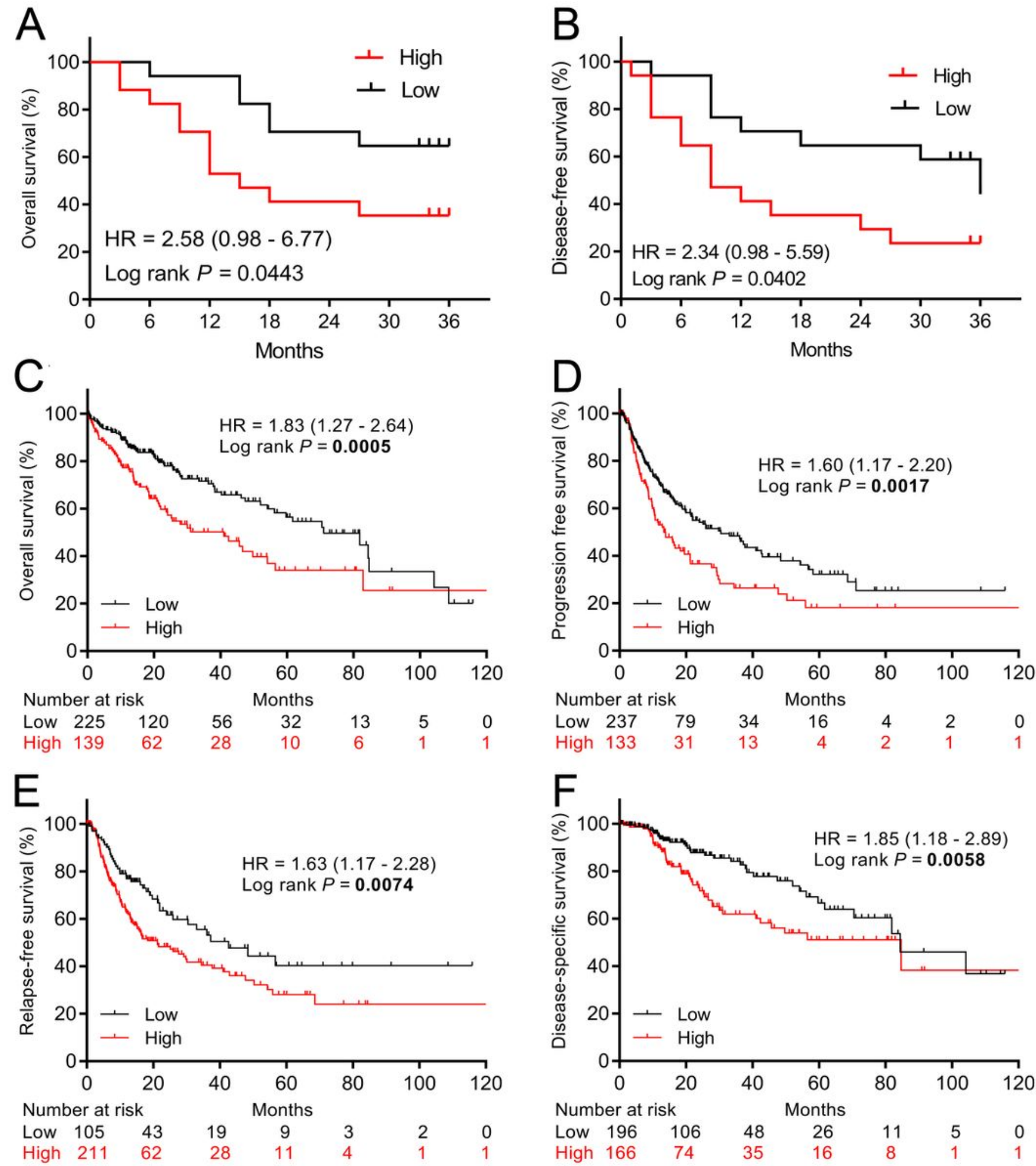

Figure 2 
SFN gene is closely associated with poor prognosis

(A, B) The overall survival (OS) and disease-free survival (DFS) of HCC patients in low or high SFN expression cohort. (C-F) The OS, progression free survival (PFS), relapse-free survival (RFS), and diseasespecific survival (DSS) of HCC cases in the Kaplan-Meier plotter online database.
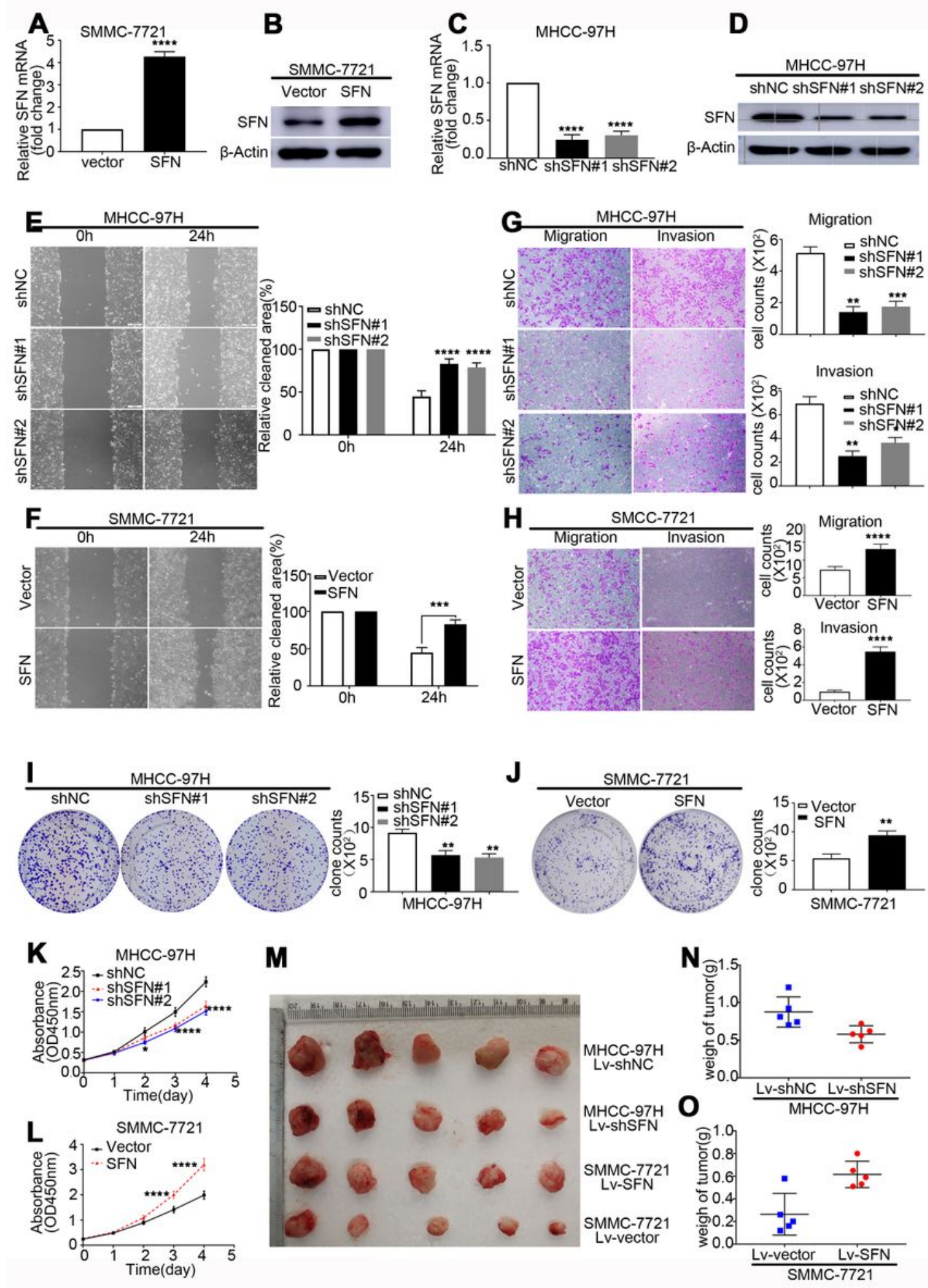

Figure 3 


\section{SFN promotes HCC cells proliferation, migration, invasion in vitro and tumor growth in vivo}

The efficiency of over-expression or knockdown of SFN was measured by RT-qPCR and western blotting in SMMC-7721 ( $A$ and B) and MHCC-97H (C and D) cell lines. The wound healing assays (E-F) and transwell migration assays (G-H) were used to explore the migratory abilities of HCC cells with upregulated or down-regulated SFN expression. (G-H) The invasion capacities of HCC cells with upregulated or down-regulated SFN expression were examined by transwell invasion assays. (I-J) The colony formation assays were utilized to investigate the proliferation abilities of HCC cells with changed SFN expression. CCK-8 assays were used to detect the proliferation capacities of MHCC-97H with SFN knockdown (K) and SMMC-7721 (L) cells with SFN over-expression. (M) The xenograft mice models analyzed the role of SFN on tumor growth, images of tumors derived from MHCC-97H with SFN knockdown or SMMC-7721 with SFN over-expression in subcutaneous of nude mice. ( $\mathbf{N}-0)$ The weigh and volume of tumor in each xenograft mice models. ${ }^{\star} \mathrm{P} \otimes 0.05,{ }^{* \star} \mathrm{P} \otimes 0.01,{ }^{* \star \star} \mathrm{P} \otimes 0.001,{ }^{\star \star \star *} \mathrm{P} \otimes 0.0001$. 
A

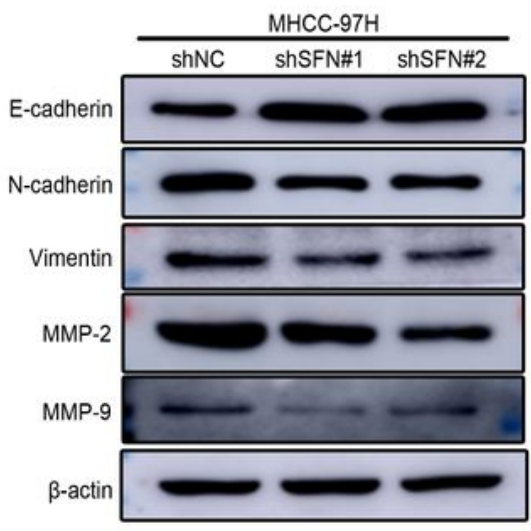

C

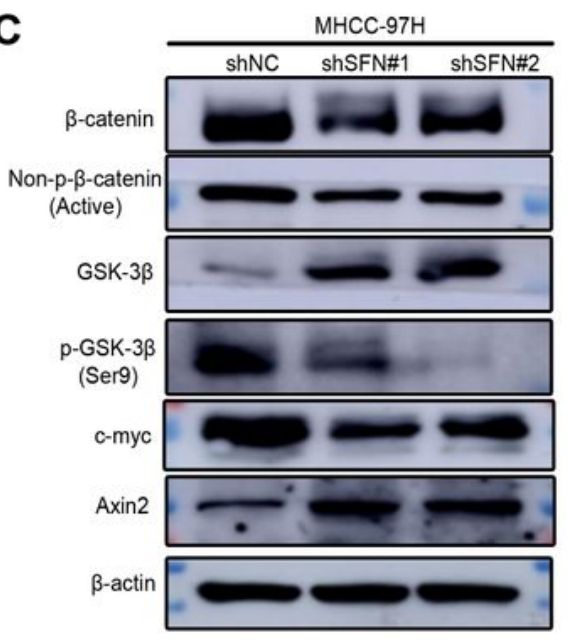

B

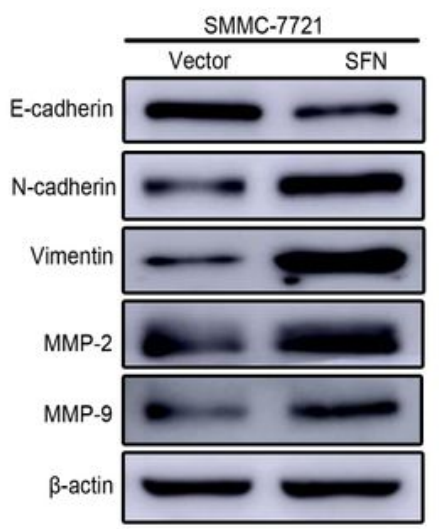

D

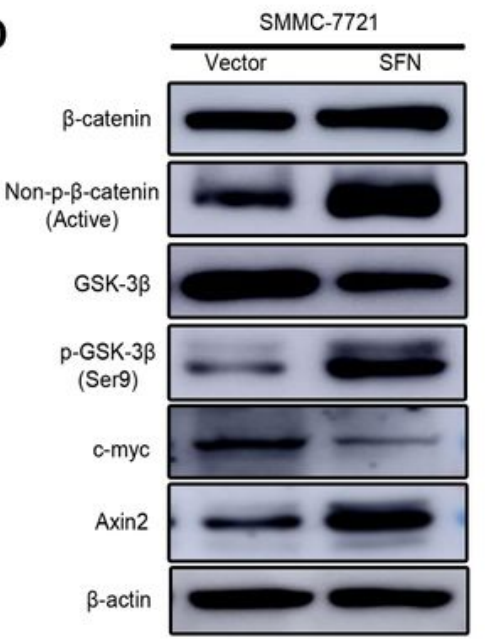

E
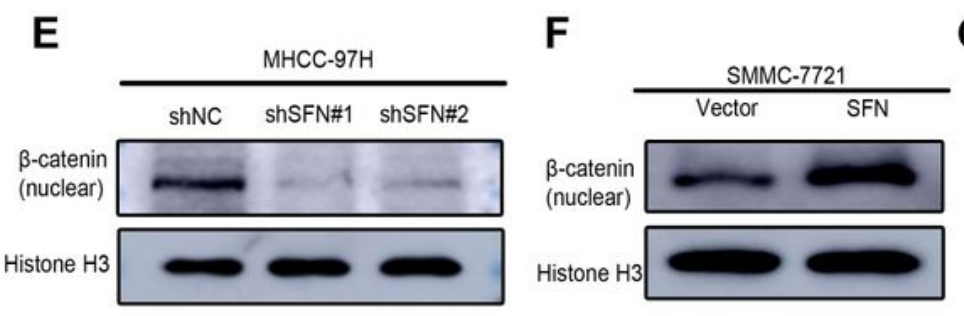

G
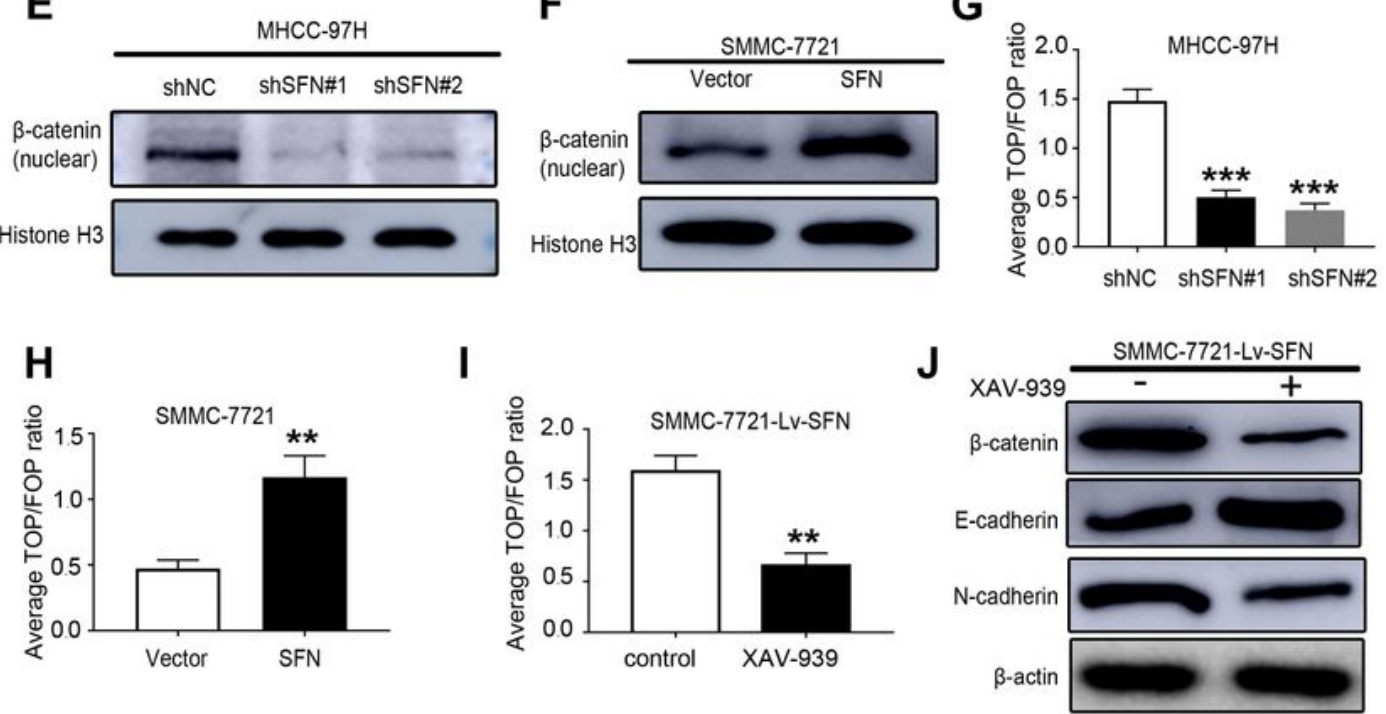

\section{Figure 4}

\section{SFN induces EMT and activates Wnt/ $\beta$-catenin signaling in HCC cells}

(A) The protein levels of E-cadherin, N-cadherin, vimentin, MMP2 and MMP9 in MHCC-97H cells infected with lentiviruses of shSFN\#1, shSFN\#2 or shNC as detected by western blot. (B)The protein levels of Ecadherin, N-cadherin, vimentin, MMP2 and MMP9 in SMMC-7721 cells infected with lentiviruses over- 
expressing SFN or vector as detected by western blot. (C) The protein levels of $\beta$-catenin, non-phospho $\beta$ -

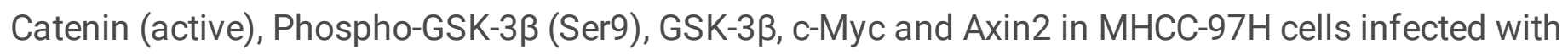
lentiviruses of shSFN\#1, shSFN\#2 or shNC as detected by western blot. (D) The protein levels of $\beta$ -

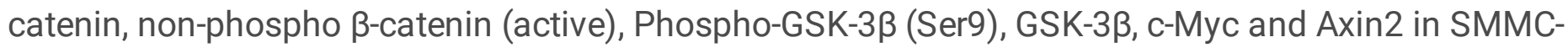
7721 cells infected with lentiviruses over-expressing SFN or vector as detected by western blot. (E)The protein level of $\beta$-catenin in nuclear of MHCC-97H cells infected with lentiviruses of shSFN\#1, shSFN\#2 or shNC as detected by western blot. (F)The protein levels of $\beta$-catenin in nuclear of SMMC-7721 cells infected with lentiviruses over-expressing SFN or vector as detected by western blot. The TOP/FOP luciferase reporter activity in MHCC-97H cells with SFN knockdown (G) and in SMMC-7721cells with SFN over-expression $(\mathrm{H})$. The TOP/FOP luciferase reporter activity $(\mathrm{I})$ and the protein levels of $\beta$-catenin, Ecadherin, N-cadherin (J) in SMMC-7721 cells with SFN over-expression treated with or without XAV-939. 